FORMATION Formation emploi

Revue française de sciences sociales

132 | octobre-décembre 2015

Enseignement supérieur et mondes économiques : de nouveaux éclairages

\title{
L'enseignement supérieur à but lucratif en France à l'aune des porosités public/privé : un état des lieux
}

For-profit higher education in France in terms of the blurry lines set the legal framework: an overview

Die privat/öffentliche Durchlässigkeit in der höheren Bildung und der gewinnorientierte Zweig in Frankreich : eine Bestandsaufnahme Las porosidades entre lo público y lo privado en la enseñanza superior y el sector con fines de lucro en Francia : un estado de la situación

\section{Aurélien Casta}

\section{OpenEdition}

\section{Journals}

Édition électronique

URL : http://journals.openedition.org/formationemploi/4561

DOI : $10.4000 /$ formationemploi.4561

ISSN : 2107-0946

Éditeur

La Documentation française

Édition imprimée

Date de publication : 30 décembre 2015

Pagination : 71-90

ISSN : 0759-6340

Référence électronique

Aurélien Casta, "L'enseignement supérieur à but lucratif en France à l'aune des porosités public/privé un état des lieux », Formation emploi [En ligne], 132 | octobre-décembre 2015, mis en ligne le 02 février 2018, consulté le 30 octobre 2020. URL : http://journals.openedition.org/formationemploi/4561 ; DOI : https://doi.org/10.4000/formationemploi.4561 


\title{
L'enseignement supérieur à but lucratif en France à l'aune des porosités public/privé : un état des lieux
}

\author{
Aurélien CASTA \\ Sociologue \\ EconomiX (CNRS - UMR 7235) - université Paris Ouest Nanterre.
}

Résumé

L'enseignement supérieur à but lucratif en France à l'aune des porosités public/ privé : un état des lieux

L'enseignement supérieur à but lucratif en France est étudié au prisme d'établissements appartenant à des groupes industriels ou financiers cherchant à valoriser leur capital. Ce secteur de l'enseignement supérieur évolue en fait dans les porosités public/privé créées par les législations du secteur. Il mobilise ainsi les différentes aides étudiantes et se construit autour de gages de qualité accessibles : Répertoire national des certifications professionnelles, autorisation de délivrer un diplôme d'Etat et reconnaissance d'établissement.

Mots clés : enseignement supérieur, enseignement privé, politique de la formation, établissement d'enseignement, financement de l'enseignement supérieur, participation de l'état, législation de l'éducation

Abstract

For-profit higher education in France in terms of the blurry lines set by the local framework: an overview

For-profit higher education is analyzed from a database of establishments owned by five private equity and multinational companies. This sector is established from the blurry lines set by laws, national commissions, agencies and ministries. First, French laws allow the students who are enrolled in the private sector to benefit from a range of benefits. Secondly, the for-profit sector is set from private-friendly regulations: the vocational registration, the rights to offer State diplomas and the acknowledgement of establishment.

Keywords : higher education, private education, training policy, educational institution, higher education funding, state participation, education legislation

Journal of Economic Literature: I 23, I 22, H 52

Traduction : Auteur 
Le ministère de l'Enseignement supérieur français a tenu, en juin 2014, à créer par décret un nouveau statut, celui d' "établissement privé d'intérêt général ». Cette nouvelle catégorie a officiellement vocation à clarifier la situation d'un secteur où il est encore d'usage, notamment à partir de la catégorie juridique d' " établissement public »" d'opposer éducation " publique » et " privée " ${ }^{2}$. Aux dires de la presse professionnelle du secteur, il s'agissait également de créer officiellement des divisions légales au sein des établissements qui n'ont pas le statut public et de distinguer, parmi eux, ce qui relève du "privé à but lucratif " (Lewandowski, 2014) ${ }^{3}$. Au-delà du conflit éducatif séculaire et très français entre public et privé (Archer, 1979 ; Deer, 2002), l'enjeu principal de telles réformes et de la position de l'enseignement supérieur à but lucratif est peut-être à rechercher davantage dans les nouvelles préoccupations des responsables nationaux et internationaux de l'enseignement supérieur. Pour ces derniers, la réflexion porte notamment sur les législations nationales, les idées et les dispositifs (fermetures/ouvertures des financements au privé, partenariats nationaux et internationaux, agences d'accréditation, commissions d'évaluation, ...) qui, en amont, soutiennent ou fragilisent l'enseignement supérieur à but lucratif et la valorisation du capital dans l'univers éducatif (Vinokur, 2004 ; Kinser et al., 2005 ; Ball, 2012).

En France, dans les sphères ministérielles de l'enseignement supérieur, le privé apparaît en effet communément accepté, ce principe n'étant associé à aucune réserve légale majeure concernant le secteur à but lucratif. Ce qui pose aujourd'hui question, c'est le "flou » et l' "ambiguïté " qui entourent l'ensemble législatif encadrant le privé (ministère de l'Education nationale [MEN], 2013 ; p. 5). Il créerait, par exemple, " une situation d'incertitude préjudiciable aux clients" (ibid). La dissolution des frontières public/privé n'est ici pas mise en question comme le montrent les rapports produits récemment aux Etats-Unis et au Royaume-Uni à la suite d'enquêtes ou de discussions informelles entre des groupes de personnalités à la croisée des différents niveaux de l'action publique (agents des services

1. Sur ce point, on pourra se reporter au Livre $7 \mathrm{du}$ Code de l'éducation. Le cas des "établissements privés" est clairement distingué de celui des "établissements publics à caractère scientifique, culturel et professionnel " (EPCSP) et des "établissements publics administratifs" (EPA). Comme l'indique le site du ministère de l'Enseignement supérieur (http://www.enseignementsup-recherche.gouv.fr/pid24558-cid49705/etablissements-d-enseignement-superieur-et-de-recherche.html ; consulté en juillet 2014), l'octroi du statut d' "établissement public " renvoie à des décrets spécifiques. Les universités, les " grands établissements " ou encore " les communautés d'établissements " jouissent du statut d'EPCSP, tandis que celui d'EPA est attribué aux Pôles de recherche et d'enseignement supérieur (PRES), aux Instituts d'études politiques de province ou encore aux Ecoles nationales supérieures d'ingénieurs. Ayant formellement le statut d'écoles " consulaires ", les écoles de commerce appartenant aux chambres de commerce et d'industrie sont couramment considérées comme des établissements "publics ", en raison du statut juridique de leur institution de rattachement.

2. Le sujet de l'enseignement supérieur privé est même explosif, comme l'ont montré les mobilisations récurrentes des étudiants, depuis la Loi Devaquet en 1986, contre la "marchandisation " de l'éducation (Legois et al., 2007).

3. Néanmoins, à la lecture du décret (n²014-635), on peine encore à voir sur quels critères ce statut et les financements de l'Etat qui l'accompagnent vont à l'avenir être attribués, le texte ne définissant que la procédure d'octroi, en l'occurrence ici sur demande de l'établissement et après consultation d'un comité $a d$ hoc. 
ministériels, directeurs d'établissements, consultants, groupes de réflexion, fondations, ...) (Barber et al., 2013 ; Presidential Innovation Lab [PIL], 2013a et b). Pour les auteurs de ces rapports, il s'agit de savoir comment les établissements à but lucratif, à l'instar de tous les établissements publics et privés (et à l'instar de leurs personnels), peuvent se spécialiser à une échelle globale selon le(s) domaine(s) d'activité dans le(s)quel ils " excellent » et sont "rentables» (ibid) : recherche fondamentale, recherche appliquée, initiation des adultes à l'entreprenariat, entretien de la main-d'œuvre du bassin économique local, etc.

Au vu de ces rapports, on peut se demander sur quels segments l'enseignement supérieur à but lucratif se spécialise et en quoi il évolue dans des porosités public/privé plus ou moins anciennes et créées par des normes et des dispositifs de nature nationale ou transnationale. Dans cette perspective, et dans la lignée de contributions sur les relations entre groupes sociaux, les conventions, les outils, les législations et les normes qui organisent la légitimation (Garcia, 2008 ; Pierru, 2012), la qualification (Gadrey, 2003 ; Karpik, 2007) et la valorisation financière (Vatin, 2009; Friot, 2012) des produits et activités, cet article vise à formuler un premier état des lieux de l'enseignement supérieur à but lucratif ainsi qu'une première analyse des normes et dispositifs autour desquels il se construit.

Pour ce faire, cet article présente les résultats d'une recherche exploratoire portant, d'une part, sur les législations françaises encadrant le privé et, d'autre part, sur les enseignes de cinq groupes d'écoles situés en France. ${ }^{4}$ A nos yeux, l'approche choisie se justifie à double titre. D'une part, elle permet de distinguer le "lucratif » au sein du privé en se concentrant sur des établissements qui ont pour point commun de se présenter comme tels, de préférer le statut de "société de capitaux " à celui d'association et de réaliser des profits. De ce point de vue, ces établissements se distinguent des autres écoles privées connues. Ces dernières organisent l'accès aux professions industrielles et commerciales et légitiment les positions sociales de celles et ceux qui les exercent (Grelon, 1986 ; Blanchard, 2012). D'autre part, cette approche rend possible d'autres recherches empiriques sur la rentabilité du secteur et la série de montages fiscaux et industriels sur laquelle il repose (Baudry, 2003), par exemple, sur les exonérations d'impôts dont bénéficient les associations partenaires des groupes ou alors sur leurs holdings qui vendent au prix fort des prestations

\footnotetext{
4. Je remercie chaleureusement Annie Vinokur pour son soutien bienveillant et exigeant tout au long de ce travail. Je suis également redevable des commentaires et critiques de Carole Sigman et Bernard Friot. Je remercie aussi les membres du pôle Sociologie politique de l'économie de l'IRISSO (Institut de recherches interdisciplinaires en sciences sociales - Université Paris Dauphine) pour l'intérêt qu'ils ont porté à ce travail. Merci enfin à l'équipe de la revue et à ses rapporteurs dont les conseils m’ont inspiré, même si je reste responsable des omissions et lacunes du texte.

5. Cette définition du "lucratif " écarte de notre champ le travailleur indépendant, qui a toujours existé depuis la fondation des premières universités (Charle et Verger, 1994), qui n’a pas d'avance en capital et qui tire sa rémunération de son travail. Par ailleurs, l'opposition lucratif/non lucratif ne recoupe pas l'opposition marchand/non marchand. Grands groupes et professions libérales écoulent tous deux leur production éducative sur le marché en échange d'un certain prix, mais les premiers relèvent de l'accumulation du capital, les secondes de la rémunération du travail.
} 
supports (communication, informatique, matériel pédagogique, ...) aux écoles des groupes sous statut non commercial. L'article s'appuie ainsi sur des données originales collectées sur les sites internet des structures étudiées mais aussi sur ceux de la presse professionnelle du secteur, des commissions nationales de qualification de l'enseignement supérieur (CEFDG CTI, RNCP) ${ }^{6}$, du portail législatif Legifrance et du Registre du commerce français. Après avoir fourni des données de cadrage sur l'enseignement supérieur privé et sur les cinq groupes étudiés, il analyse successivement en quoi leur financement et les gages de qualité qu'ils mobilisent distinguent les établissements les uns et des autres et les inscrivent dans les porosités public/privé construites par les législations du secteur.

\section{Encadré 1 - Enquête : méthode et matériaux}

Le panel regroupe 67 enseignes et 236 établissements situés sur le territoire français. L'ensemble a été sélectionné à partir des sites internet des enseignes françaises de lonis, Apax, Duke street, Bregal et Laureate. Les enseignes françaises d'Apax, de Duke street, de Bregal sont respectivement rassemblées au sein des groupes INSEEC (Institut des hautes études économiques et commerciales), Eduservices et Studialis. Les données ont été collectées en juin-juillet 2014.

Parmi les 53 enseignes pour lesquelles une date de création est annoncée, moins d'un dixième (5) a été fondé après les années 2000. Entre deux tiers et trois quart des enseignes ont ouvert leurs portes pour la première fois à Paris, un peu moins de la moitié s'étant déclinée par la suite sous la forme d'établissements sur le reste du territoire.

Toutes les enseignes pratiquent officiellement une sélection à l'entrée (concours, dossiers, entretiens, "oral de motivation », ...). Seules les enseignes dédiées en partie ou totalement à la formation initiale post-bac ont été recensées et analysées. La petite dizaine d'enseignes appartenant aux cinq groupes mais relevant totalement de la préparation au bac, des cours de soutien, de préparation aux tests de langue ou de la formation continue a été écartée de l'étude.

Ces cinq ensembles d'enseignes et d'établissements sont les plus importants aux dires de la presse professionnelle du secteur ; il est cependant difficile d'estimer exactement le nombre d'étudiants inscrits dans les établissements du panel. Les groupes lonis, Inseec, Eduservices et Studialis annoncent respectivement 17 000, 14 500, 14000 et 14500 étudiants inscrits. Le décompte effectué à partir des sites des enseignes est largement incomplet, l'information n'étant fournie que dans un tiers des cas sur les sites internet. Enfin, la recherche des enseignes sur le site internet des registres du commerce (Infogreffe) fournit un aperçu de l'entremêlement des statuts juridiques, commerciaux et associatifs, dans lequel ces groupes évoluent (cf. tableau 2). Les ensembles d'enseignes et d'établissements étudiés ici sont indistinctement dénommés « groupes », « entreprises » ou « firmes ».

6. Respectivement Commission d'évaluation des formtions et diplômes de gestion, Commission des titres d'ingénieurs, Répertoire national de la certification professionnelle. 
Ces écoles étant régulièrement soupçonnées, par le grand public, de publicité mensongère s'agissant de ses relations avec l'Etat (MEN, 2013), les informations délivrées sur les sites des enseignes au sujet de leur qualité ont été croisées à partir des textes de lois collectés sur le site Legifrance et des sites de la Commission des titres d'ingénieurs (CTI), du Répertoire national de la certification professionnelle (RNCP) et de la Commission d'évaluation des formations et diplômes de gestion (CEFDG) qui permet aux écoles de commerce de délivrer le grade de master.

Cette méthode permet de systématiquement vérifier sur des sites web institutionnels les données concernant les gages de qualité que mobilisent les enseignes (reconnaissance d'établissement, inscriptions des certifications au RNCP, autorisation de délivrer un diplôme d'Etat...).

Les données financières fournies sur les sites (frais d'inscription, partenariats avec les banques commerciales, aides mises en place pour les étudiants, ...) n'ont en revanche pas pu être vérifiées de façon aussi approfondie et rendent nécessaire la mobilisation d'autres matériaux collectés par entretiens ou consultation d'archives. Enfin, notons que d'autres groupes à but lucratif, en apparence plus petits et spécialisés, ont fait l'actualité médiatique et mériteraient sans doute d'être étudiés. On peut citer, par exemple, les groupes d'écoles français Media school group (communication et journalisme) et Novetude (santé), ainsi que les fonds d'investissement Octant Partenaire (santé), 123 Venture (informatique), Montefiore (santé), Activa (santé) et Providence equity (arts).

$\left({ }^{*}\right)$ : En octobre 2015, la presse a d'ailleurs annoncé le rachat de Studialis, détenu jusque-là par Bregal capital, par Providence equity. Au vu de son site internet, Providence equity est un fonds d'investissement américain créé en 1989 et détenant des titres de propriété et des entreprises dans plus d'une dizaine de pays et dans des domaines tels que l'éducation, les technologies de l'information, les médias et la communication.

\section{L'enseignement supérieur à but lucratif : données de cadrage et terrain d'enquête}

Comme évoqué dans l'encadré $\mathbf{1}$, cette recherche repose sur un terrain d'enquête limité à une série de 236 établissements au statut juridique privé et dans lesquels entre 50000 et 100000 étudiants seraient inscrits. Aux dires des enseignes et de la presse professionnelle du secteur, ces établissements appartiennent à cinq groupes cherchant officiellement à valoriser leur capital.

Si l'on s'intéresse à la situation qu'occupent ces écoles dans le paysage de l'enseignement supérieur français, on peut, dans un premier temps, se reporter aux statistiques ministérielles. Les données collectées et estimées offrent en effet un décompte précis du nombre d'étudiants inscrits dans le secteur privé et de leur répartition entre les filières (MEN, 2014) ${ }^{7}$. Au sein de cette population, ce sont les écoles de commerce et de comptabi-

7. Le décompte et la répartition des établissements s'avèrent moins précis et plus lacunaires que pour les étudiants (MEN, 2014 ; p. 73). Les données collectées montrent aussi un secteur "privé subventionné " par 
lité (pour près d'un tiers) puis les sections de techniciens supérieurs, les écoles d'ingénieurs, les écoles " de spécialités diverses » et celles préparant aux "fonctions sociales " qui concentrent les trois quart des effectifs (tableau 1). Les écoles étudiées ne publiant pas systématiquement leurs effectifs sur leur site web, aucune comparaison des populations étudiantes n'est en l'état possible. Néanmoins, on observe que les écoles de commerce et de comptabilité représentent plus de la moitié du panel de 67 enseignes, loin devant les écoles

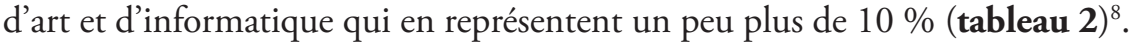

Tableau 1 : Effectifs et répartition des étudiants dans l'enseignement supérieur en France, en 2012/2013

\begin{tabular}{|c|c|c|c|}
\hline & $\begin{array}{c}\text { Etudiants (en } \\
\text { milliers) }\end{array}$ & $\begin{array}{c}\text { Part dans le secteur } \\
\text { privé }\end{array}$ & $\begin{array}{r}\text { Part dans } \\
\text { l'ensemble }\end{array}$ \\
\hline Universités & 1411,2 & & $58,7 \%$ \\
\hline $\begin{array}{l}\text { STS (sections de techniciens supérieurs) lycées } \\
\text { publics }\end{array}$ & 168,7 & & $7 \%$ \\
\hline STS lycées privés & 62 & $14 \%$ & $2,6 \%$ \\
\hline Classes préparatoires lycées publics & 69 & & $2,8 \%$ \\
\hline Classes préparatoires lycées privés & 11,1 & $2,5 \%$ & $0,5 \%$ \\
\hline Autres cursus lycées publics & 9,5 & & $0,5 \%$ \\
\hline Autres cursus lycées privés & 4,9 & $1,1 \%$ & $0,2 \%$ \\
\hline $\begin{array}{l}\text { Etablissements privés d'enseignement } \\
\text { universitaire }\end{array}$ & 29,3 & $6,7 \%$ & $1,2 \%$ \\
\hline $\begin{array}{l}\text { Grands établissements (université Paris } \\
\text { Dauphine, Science po Paris, ...) }\end{array}$ & 87,6 & & $3,7 \%$ \\
\hline Ecoles normales supérieures & 5,2 & & $0,2 \%$ \\
\hline Ecoles d'ingénieurs publics & 65,1 & & $2,6 \%$ \\
\hline Ecoles d'ingénieurs privés & 45,3 & $10,4 \%$ & $1,9 \%$ \\
\hline $\begin{array}{l}\text { Instituts nationaux polytechniques/Universités } \\
\text { de technologie }\end{array}$ & 12,8 & & $0,5 \%$ \\
\hline $\begin{array}{l}\text { Ecoles publiques de commerce, gestion et } \\
\text { comptabilité }\end{array}$ & 1 & & $0 \%$ \\
\hline $\begin{array}{l}\text { Ecoles privées de commerce, gestion et } \\
\text { comptabilité }\end{array}$ & 132,8 & $30,5 \%$ & $5,6 \%$ \\
\hline Ecoles publiques juridiques et administratives & 5,1 & & $0,2 \%$ \\
\hline Ecoles privées juridiques et administratives & 4,4 & $1 \%$ & $0,2 \%$ \\
\hline Ecoles privées de journalisme et écoles littéraires & 6,8 & $1,6 \%$ & $0,3 \%$ \\
\hline
\end{tabular}

"l'Etat" et "les collectivités locales" à hauteur respectivement de 10,5\% et 16,5\% (ibid, p. 349).

8. Les proportions sont similaires si l'on se concentre sur les 236 établissements, les écoles de commerce représentant une part légèrement plus importante, loin devant l'informatique et les métiers de la mode situés aux alentours de $10 \%$ (tableau 2). D’autres bémols doivent être apportés à cette première mesure de la représentativité du panel d'enquête. Les frontières entre filières sont, dans les faits, moins étanches. Les écoles proposent en effet régulièrement plusieurs types de certifications, n’hésitant pas, notamment, à mêler commerce, tourisme et communication. De même, sans l'afficher ouvertement, les écoles proposent fréquemment des préparations aux brevets de technicien supérieur (BTS). 


\begin{tabular}{|l|c|c|c|}
\hline Ecoles publiques paramédicales (données 2011) & 76,2 & & $3 \%$ \\
\hline Ecoles privées paramédicales (données 2011) & 24,1 & $5,5 \%$ & $1 \%$ \\
\hline $\begin{array}{l}\text { Ecoles publiques préparant aux fonctions } \\
\text { sociales (données 2011) }\end{array}$ & 1,1 & $9 \%$ & $1,6 \%$ \\
\hline $\begin{array}{l}\text { Ecoles privées préparant aux fonctions sociales } \\
\text { (données 2011) }\end{array}$ & 39,2 & & $0,7 \%$ \\
\hline $\begin{array}{l}\text { Ecoles publiques supérieures artistiques et } \\
\text { culturelles }\end{array}$ & 17 & $6,9 \%$ & $1,3 \%$ \\
\hline $\begin{array}{l}\text { Ecoles privées supérieures artistiques et } \\
\text { culturelles }\end{array}$ & 29,9 & & $0,7 \%$ \\
\hline Ecoles publiques d'architecture & 17,5 & $0,2 \%$ & $0 \%$ \\
\hline Ecoles privées d'architecture & 0,9 & & $0,1 \%$ \\
\hline Ecoles vétérinaires & 2,5 & & $0 \%$ \\
\hline Autres écoles publiques de spécialités diverses & 1 & $10,4 \%$ & $1,9 \%$ \\
\hline Autres écoles privées de spécialités diverses & 45,5 & $100 \%$ & $18,3 \%$ \\
\hline Total privé & 436,1 & & $81,7 \%$ \\
\hline Total public & 1950,8 & & $100 \%$ \\
\hline Total & 2386,9 & & \\
\hline
\end{tabular}

Source: MEN, 2014 ; p. 171.

Tableau 2 : Caractéristiques des cinq groupes d'écoles françaises investigués. Données recueillies sur les sites web des groupes propriétaires, de leurs enseignes et des registres français du commerce $(*)$

\begin{tabular}{|c|c|c|c|c|c|}
\hline $\begin{array}{l}\text { Dénomination } \\
\text { internationale }\end{array}$ & Apax partners & Bregal capital & $\begin{array}{l}\text { Duke Street } \\
\text { capital }\end{array}$ & Ionis & $\begin{array}{c}\text { Laureate } \\
\text { international } \\
\text { universities }\end{array}$ \\
\hline $\begin{array}{l}\text { Secteur d'activité et } \\
\text { volume financier des } \\
\text { propriétés détenues }\end{array}$ & $\begin{array}{l}\text { Technologies, télé- } \\
\text { communications, } \\
\text { média, distribu- } \\
\text { tion et biens de } \\
\text { consommation } \\
\text { (dont éducation } \\
\text { et santé), services } \\
\text { aux entreprises et } \\
\text { services financiers. } \\
2,5 \text { milliards } \\
\text { d'euros. }\end{array}$ & $\begin{array}{l}\text { Distribution } \\
\text { et biens de } \\
\text { consommation } \\
\text { (dont éducation } \\
\text { et santé), services } \\
\text { aux entreprises et } \\
\text { services financiers. } \\
3 \text { milliards d'euros. }\end{array}$ & $\begin{array}{l}\text { Distribution } \\
\text { et biens de } \\
\text { consommation } \\
\text { (dont éducation } \\
\text { et santé), services } \\
\text { aux entreprises et } \\
\text { services financiers. }\end{array}$ & $\begin{array}{l}\text { Enseignement } \\
\text { supérieur. }\end{array}$ & $\begin{array}{l}\text { Education. } \\
786 \text { millions } \\
\text { d'euros }\end{array}$ \\
\hline Lieux des propriétés & $\begin{array}{l}\text { France, Suisse, } \\
\text { Pays-Bas et Suède }\end{array}$ & $\begin{array}{c}\text { France et } \\
\text { Royaume-Uni }\end{array}$ & $\begin{array}{c}\text { France et } \\
\text { Royaume-Uni }\end{array}$ & France & $\begin{array}{c}29 \text { pays (Amérique, } \\
\text { Europe, Afrique et Asie) }\end{array}$ \\
\hline $\begin{array}{l}\text { Dénomination } \\
\text { internet } \\
\text { du groupe français }\end{array}$ & Inseec & Studialis & Eduservices & $\begin{array}{l}\text { Ionis education } \\
\text { group }\end{array}$ & \\
\hline $\begin{array}{l}\text { Nombre d'enseignes } \\
\text { et d'établissements }\end{array}$ & $\begin{array}{c}10 \text { enseignes } \\
21 \text { établissements }\end{array}$ & $\begin{array}{l}22 \text { enseignes } \\
36 \text { établissements }\end{array}$ & $\begin{array}{l}14 \text { enseignes } \\
117 \text { établissements }\end{array}$ & $\begin{array}{l}17 \text { enseignes } \\
\quad 54 \\
\text { établissements }\end{array}$ & $\begin{array}{l}4 \text { enseignes } \\
11 \text { établissements }\end{array}$ \\
\hline
\end{tabular}




\begin{tabular}{|c|c|c|c|c|c|}
\hline $\begin{array}{l}\text { Statut juridique des } \\
\text { enseignes }\end{array}$ & $\begin{array}{l}2 \text { AS/SAS } \\
6 \text { ND } \\
\text { 2SARL }\end{array}$ & $\begin{array}{l}4 \text { AS/SAS } \\
8 \mathrm{ND} \\
10 \mathrm{SAS}\end{array}$ & $\begin{array}{l}1 \text { ND } \\
3 \text { SA } \\
9 S A S \\
1 \text { SARL } \\
\end{array}$ & $\begin{array}{c}7 \mathrm{AS} \\
1 \mathrm{AS} / \mathrm{GIE} \\
1 \mathrm{AS} / \mathrm{SARL} \\
2 \mathrm{AS} / \mathrm{SARL} / \mathrm{SAS} \\
2 \mathrm{AS} / \mathrm{SAS} \\
\\
3 \mathrm{ND} \\
1 \mathrm{SA}\end{array}$ & $\begin{array}{l}1 \text { AS } \\
2 \text { AS/SAS } \\
1 \text { ND }\end{array}$ \\
\hline Effectifs annoncés & 14500 & 14500 & 14000 & 17000 & ND \\
\hline $\begin{array}{l}\text { Domaine d'activité } \\
\text { des enseignes (et } \\
\text { des établissements) } \\
\text { situées en France? }\end{array}$ & $\begin{array}{l}1 \text { ART (ND) } \\
7 \text { COM (17) } \\
2 \mathrm{SOC}(4)\end{array}$ & $\begin{array}{c}7 \text { ART (9) } \\
10 \text { COM (18) } \\
2 \text { COMMU (2) } \\
3 \text { INF0 (6) }\end{array}$ & $\begin{array}{c}10 \text { COM (72) } \\
1 \text { COMMU (7) } \\
2 \text { DIV (16) } \\
1 \text { MOD (22) }\end{array}$ & $\begin{array}{l}7 \text { COM (26) } \\
1 \text { DIV (1) } \\
4 \text { INFO (19) } \\
3 \text { INGE (6) } \\
1 \text { MOD (1) } \\
1 \text { SOC (1) }\end{array}$ & $\begin{array}{l}2 \text { COM (9) } \\
1 \text { INF0 (1) } \\
1 \text { INGE (1) }\end{array}$ \\
\hline
\end{tabular}

Source : Enquête.

Clés de lecture:

Domaines:

ART : métiers de l'art et du cinéma

SOC: préparations aux concours de la santé et du social

COM : commerce, ressources humaines, gestion, finance, marketing et comptabilité

COMMU : métiers de la communication, de la publicité et du journalisme

DIV : 1 biotechnologie, 2 tourisme.

INF0 : informatique et métiers du numérique

INGE : école d'ingénieurs

MODE : métiers du luxe et de la mode

Statut juridique:

AS : association

ND: donnée indisponible

SA: société anonyme

SARL : société anonyme à responsabilité limitée

SAS : société par actions simplifiée

GIE : groupe d'intérêt économique

$\left(^{*}\right)$ : Toute société est tenue de faire enregistrer auprès du greffier du Tribunal de commerce ses comptes annuels ainsi que ses statuts constitutifs, ses cessions de parts sociales ou de biens immobiliers, etc.

Plus généralement, les tableaux $\mathbf{1}$ et $\mathbf{2}$ montrent que privé et lucratif proposent d'emblée une palette variée de domaines disciplinaires en partie similaire à celle du secteur public. Pour autant, les firmes, les fonds d'investissement et les enseignes qui rassemblent les établissements étudiés semblent aussi chercher, sous certains aspects, à se distinguer et à valoriser une identité qui leur est propre.

Ils se présentent ainsi la plupart du temps sur leur site web et dans la presse comme des groupes " privés». De même, ils s'affichent ouvertement comme des entrepreneurs se construisant autour d'une "vocation ", dotés de "valeurs ", en prise avec les réalités du "marché du travail " et recherchant l' "innovation pédagogique ". Un faisceau d'indices de nature institutionnelle, juridique et comptable laisse enfin à penser qu'il s'agit aussi de groupes cherchant à tirer des profits des propriétés qu'ils détiennent dans l'enseignement 
supérieur. Malgré l'invisibilisation juridique dont les établissements étudiés ici font l'objet et malgré le secret des affaires qui les entoure (Kinser et al., 2005), on peut en effet formuler l'hypothèse qu'ils relèvent de l'enseignement supérieur à but lucratif car ils appartiennent à cinq groupes qui rassemblent au moins une des trois conditions suivantes : (i) les firmes et les fonds auxquels ils appartiennent sont présentés ou se présentent sur leur site internet et/ou dans la presse professionnelle du secteur comme des organisations recherchant la rentabilité ; (ii) les déclinaisons des groupes en France (Inseec, Studialis, Ionis, Eduservices) sont enregistrées en tant que sociétés commerciales ${ }^{9}$; (iii) les déclarations des groupes français au registre du commerce font état des profits annuels réalisés.

Parmi les cinq groupes choisis, seuls Duke street et Ionis réunissent l'ensemble des critères. La firme Ionis a été fondée en France. Elle réunit 17 enseignes et 54 établissements. Présentée comme le dernier groupe d'écoles à résister aux propositions des fonds anglosaxons, la firme fait régulièrement l'actualité française de l'enseignement supérieur à but lucratif et est considérée comme l'une de celles capables de répondre aux exigences de rentabilité des détenteurs de capitaux ("entre 8 et $10 \% »)$ (Monod, 2011) (i). Comme le montre le site Infogreffe, Ionis est enregistrée comme une société commerciale (ii) et affiche, depuis 2010, des résultats bénéficiaires de l'ordre de plusieurs centaines de milliers d'euros (iii). Vient ensuite le groupe Apax partner. Fondé en France et au Royaume-Uni, le groupe détient 12 enseignes et 21 établissements qu'il a rachetés en 2013 à la firme nord-américaine Career education, un groupe coté spécialisé dans l'éducation. Concernant Apax, la presse professionnelle française et le site du fonds permettent de constater ses visées lucratives (i). Le groupe français qu'il a racheté, Inseec, est inscrit en tant que société commerciale sous la forme d'une entité (ii) dont les comptes n'ont pas été déposés systématiquement.

Apax Partner présente une apparence similaire à celle de Duke Street et Bregal capital qui détiennent, depuis plusieurs années, des ensembles d'établissements d'enseignement supérieur français. Selon la presse professionnelle du secteur et leurs sites internet, ces fonds, créés au Royaume-Uni, se distinguent des groupes industriels car ils appartiennent à un petit groupe de propriétaires détenant des parts dans un large éventail d'activités à but lucratif (i). Duke street capital détient ainsi, depuis 2010, au sein de son large portefeuille, 14 enseignes et 117 établissements dans l'enseignement supérieur français. Le groupe dont il est propriétaire, Eduservices, est enregistré en tant que société commerciale (ii) et annonçait, en 2012, un bénéfice de près d'un million d'euros (iii) ${ }^{10}$. De son côté, Bregal capital détient 21 enseignes réparties en 36 établissements regroupés au sein de Studialis. Si la firme en question est inscrite en tant que société commerciale (ii), elle a fait le choix de ne pas déposer ses comptes annuels au cours des quatre derniers exercices.

9. Alors qu'on voit dans le tableau 2 que leurs enseignes hésitent entre statut associatif et statut commercial. 10. Dans un entretien paru le 19 janvier 2011 dans Educpros, Philippe Grassaud, le président d'Eduservices, évoquait " un taux de rentabilité [demandé] classique autour de $8 \%$ \%. Une nuance à apporter toutefois : l'exercice 2013 aurait enregistré une perte de près d'un demi-million d'euros. 
Le dernier des cinq groupes présente une forme plus atypique. Créé aux Etats-Unis, Laureate est un fonds d'investissement spécialisé dans l'éducation. Il appartient officiellement à un petit groupe de propriétaires et à une organisation non gouvernementale (ONG) évoluant dans le giron de la Banque Mondiale, IFC (Elledge, 2014) ${ }^{11}$. Classé comme un groupe à but lucratif par la presse française étudiée, Laureate international universities se présente régulièrement comme un "réseau " de 80 établissements d'enseignement supérieur. Bien quaucune entreprise ne représente le fonds en France, et que les liens qui l'unissent aux écoles du territoire semblent plus flous que pour les autres groupes, la presse présente régulièrement Laureate comme le propriétaire de quatre enseignes et onze établissements français.

Ces données sur les groupes détenant des établissements d'enseignement supérieur, en France, laissent apparaître des ensembles d'entreprises visant clairement la rentabilité financière mais qui, au final, affichent, un peu à la manière des établissements sous tutelle de l'Etat, une large palette de cursus d'enseignement. Nous proposons par la suite d'explorer plus en détail cette ambiguïté, de voir la façon dont elle est utilisée par les différents groupes et d'expliquer l'entremêlement des financements et des gages de qualité que pratiquent les établissements à but lucratif par les porosités public/privé induites ou tolérées par les législations du secteur.

\section{Un financement en partie socialisé}

Pour peu que l'on adopte une approche systémique du financement de l'enseignement supérieur en s'intéressant également, à l'instar des sites internet des établissements, aux ressources distribuées aux étudiants, plusieurs enseignements peuvent être tirés quant au mode de financement sur lequel reposent les écoles françaises à but lucratif. ${ }^{12}$

Le décret n56-931 de 1956 et la Loi du 18 mars 1880 sur la liberté de l'enseignement supérieur fixent les cadres généraux de l'enseignement supérieur privé (MEN, 2013). Deux statuts coexistent ainsi aujourd'hui dans le Code de l'éducation : "L'enseignement tech-

11. IFC a récemment fait l'actualité car il s'est approprié Coursera, une plateforme de Princeton qui a dernièrement inauguré une forme d'enseignement en ligne, les « massive open online courses " (MOOC), qui ne permet pas d'obtenir de certifications des établissements mais qui rend possible l'accès, simultané et à un prix plus ou moins élevé, d'une infinité d'individus à des vidéos d'enseignement et à des services complémentaires. La principale activité d'IFC consiste, à partir d'un capital de près de 800 millions d'euros, à prendre le contrôle de groupes et d'établissements éducatifs ayant, aux dires de l'un de ses représentants, un " haut impact sur le développement économique " et à leur imposer des objectifs de "rentabilité commerciale " (" commercial returns") au nom du fait qu' "ils sont un gage de stabilité" (cité par Elledge, op. cit.).

12. Une étude approfondie des formes de financement des établissements à but lucratif nécessiterait probablement un savoir-faire de comptable et surtout un accès à l'ensemble de la documentation (bilans, compte de résultats, ...) produite par les différentes enseignes. 
nique " et l' "enseignement supérieur libre ", catégorie utilisée dans un premier temps pour dénommer une dizaine d'établissements à visées généraliste et confessionnelle ${ }^{13}$. Le décret $\mathrm{n}^{\circ}$ 56-931 rend explicitement possible un subventionnement direct ou indirect des établissements qui n'appartiennent pas à l'Etat. Tout établissement peut ainsi prétendre à l'octroi de «subventions » ou de « bourses » de l'Etat. Rien n'empêche formellement les collectivités locales (régions, départements, municipalités) d'en faire de même. Plusieurs lois (comme par exemple la loi $\mathrm{n}^{\circ}$ 48-472), décrets $\left(\mathrm{n}^{\circ}\right.$ 48-2006) et arrêtés (notamment celui du 27 novembre 1955), adoptés après-guerre, permettent aux étudiants inscrits dans l' "enseignement supérieur technique " de bénéficier des bourses d'Etat et des prestations " maladie " du régime général de la sécurité sociale. Formellement, ce sont uniquement les étudiants inscrits dans les établissements reconnus par l'Etat (encadré 2) qui peuvent bénéficier de ce type de prestations, mais l'absence de texte législatif clair et le peu de contrôle effectivement mis en place par le ministère et ses services laissent la porte en partie ouverte aux établissements non reconnus (MEN, 2013).

De même, aucun texte n'exclut les étudiants des enseignements supérieurs techniques et libres du bénéfice des allocations logement versées par le régime "famille " de la sécurité sociale $^{14}$. Pour les établissements à but lucratif, ce cadre légal ouvre la voie à un modèle de financement reposant, pour une bonne partie, sur les fonds que l'Etat, les collectivités et la sécurité sociale socialisent à l'occasion de la collecte des cotisations assises sur les salaires et des impôts sur la valeur ajoutée ou le revenu des ménages.

Au regard de la communication qui est faite autour de ces fonds socialisés sur les sites des enseignes, ils constituent l'un des piliers du financement de l'enseignement supérieur à but lucratif (tableau 3). Comme on peut s'y attendre, ces écoles demandent aux étudiants des frais d'inscription élevés. Mais elles les encouragent aussi à recourir à des prêts étudiants distribués par le biais de partenariats avec les banques commerciales et à solliciter les bourses internes qu'elles offrent. La quasi-totalité des 46 enseignes (43) pour lesquelles la donnée est disponible annonce ainsi, pour l'année 2014/2015, des frais d'inscription compris entre 3800 et 14900 euros. Plus généralement, deux tiers des enseignes (40) déclarent proposer des prêts à taux " avantageux " par le biais de conventions avec les banques commerciales. Seul un petit cinquième d'entre elles (appartenant essentiellement au groupe Ionis) et un quart d'entre elles (20) font état respectivement de services dédiés à l'emploi étudiant et de bourses d'établissement distribuées au mérite ou sur critères sociaux.

13. Le décret concerne toujours uniquement l' "enseignement technique » qui, à l'époque, était défini comme un enseignement formant aux métiers du commerce ou de l'industrie. A part la visée des enseignements, rien n'exclut a priori l' "enseignement supérieur libre " de ce décret. Sans clarifier réellement les frontières respectives des deux secteurs (MEN, 2013), le ministère a toutefois commencé à produire, depuis le début des années 2000, des textes de lois officiellement dédiés au secteur "libre ", par exemple l'arrêté du 21 octobre 2003 portant création du comité consultatif pour l'enseignement supérieur privé.

14. Aucun texte législatif spécifique n’a pu être retrouvé en la matière. Le Code de la sécurité sociale prévoit simplement certaines règles pour calculer le montant auquel les « étudiants » ont droit. En 2012/2013, environ 1,5 milliards d'euros d'allocations logement étaient versés aux étudiants (MEN, 2014). 


\section{Encadré 2 : Reconnaissance de l'établissement, Grade de master, Titre d'ingénieur, Visa et Certifications enregistrées au RNCP}

La "reconnaissance » de l'établissement privé par le ministère de l'Education nationale est accordée à la suite d'une présentation, par l'école, des «plans d'études et programmes» (Loi Astier de 1919 sur l'enseignement technique).

L'autorisation de délivrer le grade de master ou le titre d'ingénieur (lui-même conférant le grade de master) est délivrée aux écoles privées à la suite de deux procédures d'évaluation par la Commission des titres d'ingénieurs (CTI) et par la Commission d'évaluation des formations et diplômes de gestion (CEFDG). Ces commissions formulent des avis d'autorisation examinées par le Conseil national de l'enseignement supérieur et de la recherche (CNESER) qui émet lui-aussi un avis. Les avis de la CEFDG et de la CTI sont en général suivis par le ministère. L'autorisation est limitée dans le temps mais les deux certifications sont a priori de même nature que les masters délivrés par les universités publiques.

La pratique, par le ministère de l'Education nationale du « visa » de certifications proposées par les établissements privés d'enseignement supérieur date de plusieurs décennies. Les visas sont attribués à la suite de demandes formulées par les écoles et comprenant une présentation de l'établissement, de ses programmes, de ses examens et des conditions d'obtention des certificats.

L'inscription des certificats au Répertoire nationale de la certification professionnelle (RNCP) est issue de la loi de 1971 ( $n^{\circ}$ 71-577) et est ouverte aux « établissements qui ne sont pas placés sous le contrôle du ministre de l'Education nationale » (art. 8). La mise à jour du répertoire est la mission essentielle de la Commission nationale de la certification professionnelle (CNCP), un organisme sous tutelle du ministère de l'Emploi. Ce dernier a la responsabilité de valider l'inscription des certifications d'établissements au sein des cinq niveaux du RNCP. Ces niveaux de formation sont équivalents, dans les nomenclatures des emplois, à différentes certifications proposées par l'enseignement public et les universités telles que le brevet d'études professionnelles (niveau 5), le brevet professionnel (niveau 4), le DUT ou le BTS (niveau 3 ; respectivement diplôme universitaire de technologie et brevet de technicien supérieur), la licence (niveau 2) ou le master (niveau 1). Cette inscription se fait sur demande auprès de la Commission qui prend principalement sa décision en fonction de l'insertion professionnelle des anciennes cohortes de diplômés, au nom de sa " mission » originelle "d'information et d'orientation » des étudiants et des employeurs (art. 3 de la loi $n^{\circ} 71-577$ ).

Tableau 3 : Financements étudiants annoncés par les enseignes des cinq groupes d'écoles françaises investigués. Données recueillies sur les sites web des enseignes (sur un total de 67 enseignes)

\begin{tabular}{|c|c|c|c|c|c|}
\hline Dénomination internationale & Apax partners & Bregal capital & $\begin{array}{l}\text { Duke Street } \\
\text { capital }\end{array}$ & Ionis & $\begin{array}{c}\text { Laureate } \\
\text { international } \\
\text { universities }\end{array}$ \\
\hline $\begin{array}{l}\text { Dénomination internet du } \\
\text { groupe français }\end{array}$ & Inseec & Studialis & Eduservices & $\begin{array}{c}\text { lonis education } \\
\text { group }\end{array}$ & \\
\hline $\begin{array}{l}\text { Nombre d'enseignes et } \\
\text { d'établissements }\end{array}$ & $\begin{array}{l}10 \text { enseignes } \\
21 \text { établissements }\end{array}$ & $\begin{array}{l}22 \text { enseignes } \\
36 \text { établissements }\end{array}$ & $\begin{array}{l}14 \text { enseignes } \\
117 \text { établissements }\end{array}$ & $\begin{array}{c}17 \text { enseignes } \\
54 \\
\text { établissements }\end{array}$ & $\begin{array}{c}4 \text { enseignes } \\
11 \\
\text { établissements }\end{array}$ \\
\hline $\begin{array}{l}\text { Montant des frais d'inscription } \\
\text { (moyenne et extrêmes }\left({ }^{*}\right) \text { ) }\end{array}$ & $\begin{array}{c}9179 \\
(850-33600) \\
1 \mathrm{ND}\end{array}$ & $\begin{array}{c}9206 \\
(4350-29500) \\
9 \text { ND }\end{array}$ & $\begin{array}{c}5453 \\
(3850-7500) \\
9 \text { ND }\end{array}$ & $\begin{array}{c}5992 \\
(0-9200) \\
2 N D\end{array}$ & $\begin{array}{c}8044 \\
(5000-12000) \\
0 N D\end{array}$ \\
\hline
\end{tabular}




\begin{tabular}{|l|c|c|c|c|c|}
\hline $\begin{array}{l}\text { Nombre d'enseignes } \\
\text { annonçant un partenariat avec } \\
\text { les banques commerciales }\end{array}$ & 5 & 12 & 6 & 14 & 3 \\
\hline $\begin{array}{l}\text { Nombre d'enseignes } \\
\text { annonçant des bourses } \\
\text { d'établissement }\end{array}$ & 5 & 4 & 0 & 9 & 2 \\
\hline $\begin{array}{l}\text { Nombre d'enseignes } \\
\text { annonçant les bourses d'Etat }\end{array}$ & 2 & 3 & 0 & 6 & 2 \\
\hline $\begin{array}{l}\text { Nombre d'enseignes } \\
\text { annonçant l'affiliation à la } \\
\text { sécurité sociale }\end{array}$ & 3 & 2 & 3 & 5 & 2 \\
\hline $\begin{array}{l}\text { Nombre d'enseignes } \\
\text { annonçant les allocations } \\
\text { logement }\end{array}$ & 3 & 1 & 0 & 7 & 3 \\
\hline $\begin{array}{l}\text { Nombre d'enseignes } \\
\text { annonçant les bourses des } \\
\text { collectivités locales }\end{array}$ & 3 & 2 & 0 & 10 & 2 \\
\hline $\begin{array}{l}\text { Nombre d'enseignes } \\
\text { annonçant les contrats } \\
\text { d'apprentissage ou de } \\
\text { professionnalisation }\end{array}$ & 5 & 14 & 14 & 3 & 3 \\
\hline
\end{tabular}

Source : Enquête

Clé de lecture:

ND Donnée indisponible

$\left.{ }^{*}\right)$ : La plupart des enseignes annonçant une demi-douzaine de prix différents pour les certifications qu'elles proposent, la moyenne générale a été estimée à partir de la moyenne des prix extrêmes proposés par chaque école. Les extrêmes n'ont quant à eux pas été recalculés. Duke Street se distingue par le peu de publicité faite sur ce point et IONIS par les prix relativement bas de ses formations.

Aux côtés de ces fonds privés, hormis les enseignes du groupe Duke Street qui semblent tout miser sur l'alternance, une publicité variée est faite aux fonds socialisés mis à la disposition des étudiants. Ainsi, 13 enseignes rappellent que leurs étudiants peuvent prétendre aux bourses d'Etat et 15 qu'ils peuvent s'affilier au régime maladie de la sécurité sociale $^{15}$. En outre, 14 enseignes font état de l'accès possible de leurs étudiants aux allocations logement et 17 évoquent l'accès aux bourses des conseils régionaux, généraux et des municipalités pouvant éventuellement être mises en place.

Les études en alternance par le biais des contrats de professionnalisation ou de contrats d'apprentissage sont elles aussi proposées dans plus de la moitié des cas (39). S'adressant principalement aux jeunes âgés de moins de 25 ans en formation initiale, ce type d'études et de contrats permet formellement à la personne de continuer ses études un certain nombre d'heures par an et d'être employée à un salaire exonéré de cotisations sociales et équivalent à deux tiers ou $85 \%$ du SMIC net (salaire minimum interprofessionnel de croissance). Les fonds socialisés de la formation continue et de l'apprentissage, en grande partie contrôlés par les employeurs et ouverts formellement

15. Il en va de même pour 15 enseignes membres du réseau Erasmus qui annoncent la possibilité de bénéficier des bourses du même nom. 
à n'importe quel type d'établissement d'enseignement supérieur ${ }^{16}$, bénéficient eux aussi d'une publicité systématique. D'une part, pour inciter les personnes à faire financer leur formation par les organismes paritaires collecteurs agréés (OPCA). D'autre part, pour inciter les employeurs à verser aux établissements à but lucratif leur taxe d'apprentissage obligatoire.

Ces éléments factuels et législatifs laissent ainsi à penser que les établissements à but lucratif se construisent sur des modèles de financement reposant à la fois sur les contributions des étudiants et de leurs familles, leur endettement auprès des banques commerciales, mais aussi sur une série de fonds socialisés relevant traditionnellement des services de l'Etat, des collectivités locales, de la sécurité sociale et de la formation continue.

\section{Des relations ambigües avec les législations, les services et les commissions de l'Etat}

On pourrait s'attendre, d'une part, à ce que les groupes d'enseignement supérieur à but lucratif aient tendance, pour faire état de leurs qualités, à se tourner par mimétisme vers les accréditations fournies par le secteur privé et que, d'autre part, les services de l'Etat, au nom de principes politiques et de contraintes organisationnelles, ainsi que les universités et les "grandes écoles ", au nom d'exigences académiques, se gardent de transférer leurs pouvoirs et leurs qualités de certificateurs aux établissements à but lucratif. Or, lorsque l'on s'intéresse aux principaux gages de qualité fournis sur leurs sites par les 67 enseignes, on s'aperçoit tout d'abord que seules 18 d'entre elles font référence aux agences d'accréditation ${ }^{17}$. Bien que peu d'entre elles soient officiellement accréditées par ces agences, une partie, en général des écoles de commerce, fait état de son appartenance à ces associations internationales et des processus de qualifica-

16. L'organisation et le mode de financement de la formation continue ont ainsi largement accompagné, depuis les années 1970, un essor des entreprises "privées» (Dubar, 2004). De son côté, la taxe d'apprentissage est officiellement dédiée à des "formations technologiques et commerciales".

17. Duke Street se distingue à nouveau dans ce paysage en faisant particulièrement référence à ces organismes privés (à neuf reprises; contre trois pour Ionis, trois pour Apax, deux pour Bregal et une pour Laureate). Les plus citées sont l'Association to Advance Collegiate Schools of Business (AACSB) et la European Federation for Management developement (EFMD). Elles sont respectivement basées aux Etats-Unis et en Belgique et comptent entre 800 et 1400 membres. L'ensemble des membres n'est pas systématiquement accrédité. Les établissements membres doivent s'engager dans un processus qui a pour caractéristiques d'être "non gouvernemental", "volontaire » et de reposer à la fois sur une "évaluation externe " et une " auto-évaluation" des établissements, des personnels, des programmes d'enseignement et de leurs stratégies de développement. Trois des 67 enseignes, appartenant toutes à Duke street, font également appel à la norme ISO, l'accréditation standard et traditionnelle des biens et services, produite par l'International Organisation for Standardization. Les enseignes étudiées font enfin, sur leurs sites, peu référence aux classements publiés régulièrement par la presse professionnelle du secteur. 
tion dans lesquels elles sont engagées. En réalité, les enseignes étudiées apparaissent bien plus proches de l'Etat français que de ces agences internationales (tableau 4). Ce constat s'explique notamment par l'état des législations existantes et les statuts actuels des enseignements supérieurs "libre " et " technique (MEN, 2013).

Tableau 4 : Reconnaissance de l'établissement, autorisation de délivrer un diplôme d'Etat et certifications inscrites au RNCP annoncées par les enseignes des cinq groupes d'écoles françaises investigués ${ }^{*}$ ).

\begin{tabular}{|c|c|c|c|c|c|}
\hline $\begin{array}{l}\text { Dénomination } \\
\text { internationale }\end{array}$ & Apax partners & Bregal capital & $\begin{array}{l}\text { Duke Street } \\
\text { capital }\end{array}$ & Ionis & $\begin{array}{c}\text { Laureate } \\
\text { international } \\
\text { universities }\end{array}$ \\
\hline $\begin{array}{l}\text { Dénomination internet } \\
\text { du groupe français }\end{array}$ & Inseec & Studialis & Eduservices & $\begin{array}{l}\text { Ionis education } \\
\text { group }\end{array}$ & \\
\hline $\begin{array}{l}\text { Nombre d'enseignes et } \\
\text { d'établissements }\end{array}$ & $\begin{array}{c}10 \text { enseignes } \\
21 \text { établissements }\end{array}$ & $\begin{array}{c}22 \text { enseignes } \\
36 \text { établissements }\end{array}$ & $\begin{array}{c}14 \text { enseignes } \\
117 \text { établissements }\end{array}$ & $\begin{array}{c}17 \text { enseignes } \\
54 \text { établissements }\end{array}$ & $\begin{array}{c}4 \text { enseignes } \\
11 \text { établissements }\end{array}$ \\
\hline $\begin{array}{l}\text { Reconnaissance } \\
\text { d'établissement }\end{array}$ & 2 & 2 & 3 & 4 & 1 \\
\hline $\begin{array}{l}\text { Autorisation de délivrer } \\
\text { un diplôme d'Etat }\end{array}$ & $\begin{array}{c}1 \text { BTS } \\
\\
1 \mathrm{MAS} / \mathrm{VB} 5 \\
7 \mathrm{SCE} \\
\text { 1VB4 }\end{array}$ & $\begin{array}{c}6 \mathrm{BTS} \\
1 \mathrm{DCG} \\
1 \mathrm{DCG} / \mathrm{DSCG} \\
1 \mathrm{MAS} / \mathrm{VB} 5 \\
13 \mathrm{SCE}\end{array}$ & $\begin{array}{c}6 \mathrm{BTS} \\
2 \mathrm{BTS} / \mathrm{DCG} \\
1 \mathrm{BTS} / \mathrm{DCG} / \mathrm{DSCG} \\
5 \mathrm{SCE}\end{array}$ & $\begin{array}{c}2 \text { BTS } \\
1 \mathrm{DCG} / \mathrm{DSCG} \\
1 \mathrm{MAS} / \mathrm{VB} 5 \\
10 \mathrm{SCE} \\
3 \mathrm{TI}\end{array}$ & $\begin{array}{c}1 \mathrm{MAS} / \mathrm{VB} 5 \\
1 \mathrm{SCE} \\
1 \mathrm{TI}\end{array}$ \\
\hline $\begin{array}{l}\text { Certifications inscrites } \\
\text { au RNCP }\end{array}$ & $\begin{array}{r}2 \mathrm{RNCP} 2 \\
8 \mathrm{SCl}\end{array}$ & $\begin{array}{c}5 \text { RNCP1 } \\
7 \text { RNCP1/RNCP2 } \\
1 \text { RNCP1/2/3 } \\
1 \text { RNCP2 } \\
1 \text { RNCP2/RNCP3 } \\
2 \text { RNCP3 } \\
5 \text { SCl }\end{array}$ & $\begin{array}{c}3 \text { RNCP1/RNCP2 } \\
3 \text { RNCP1/2/3 } \\
6 \text { RNCP2 } \\
2 \text { RNCP2/RNCP3 }\end{array}$ & $\begin{array}{c}7 \text { RNCP1 } \\
1 \text { RNCP1/RNCP2 } \\
1 \text { RNCP2 } \\
8 \mathrm{SCl}\end{array}$ & $\begin{array}{l}1 \text { RNCP1 } \\
\text { 3SCl }\end{array}$ \\
\hline
\end{tabular}

Source : Enquête.

Clés de lecture:

BTS : Brevet de technicien supérieur

TI: Titre d'ingénieur

DCG : Diplôme de comptabilité et de gestion

SCE : Etablissement dont les formations ne mènent à aucun diplôme d'Etat

DSCG : Diplôme supérieur de comptabilité et de gestion

SCI : Etablissement dont les certifications ne sont pas inscrites au RNCP (Répertoire national des certifications professionnelles)

MAS: Grade de Master

VB4 : Certification visée à $\mathrm{Bac}+4$

VB5 : Certification visée à $\mathrm{Bac}+5$

RNCP1 : Certification inscrite au RNCP niveau 1

RNCP2 : Certification inscrite au RNCP niveau 2

RNCP3 : Certification inscrite au RNCP niveau 3.

(*) Données recueillies sur les sites web des enseignes et vérifiées sur les sites web Legifrance, RNCP, CTl et CEFDG. Une demi-douzaine d'enseignes n'ont pas été prises en compte. Elles font état sur leur site de qualifications dont on n'a pas pu retrouver la trace sur les sites institutionnels. 
Douze enseignes sont ainsi officiellement reconnues par le ministère de l'Education nationale. De même, la moitié des enseignes (31) offre des voies d'accès aux diplômes d'Etat. En la matière, tous les diplômes et toutes les stratégies ne se valent pas. Plus des deux tiers (22) d'entre elles ne font que proposer des préparations et une inscription aux concours nationaux des études de comptabilité et des sections de techniciens supérieurs (STS). Le tiers restant (9) délivre directement des diplômes du ministère de l'Education nationale ou de l'Enseignement supérieur. Une partie a accès au "visa " de ces diplômes par le ministère. Une autre partie a accès à deux plus prestigieuses autorisations formellement octroyées au cas par cas par le ministère par le biais d'arrêtés législatifs : le titre d'ingénieur et le "grade " universitaire de master ${ }^{18}$. Ce type de garantie tire notamment son plus grand prestige de ses références à "l'excellence académique " et des procédures d'évaluation des activités de recherche mises en place respectivement par la CTI (Grelon, 1986) et par la CEFDG $^{19}$ (Blanchard, 2012). Les quelques écoles autorisées à délivrer ces diplômes sont en général présentées comme des " têtes de gondole " par les groupes auxquels elles appartiennent.

Lorsque les diplômes d'Etat sont délivrés, c’est aussi dans le cas de conventions signées avec les écoles d'Etat et les universités ${ }^{20}$. Les conventions portent sur des ressources variées (autorisation de délivrer des licences et des masters mais aussi accès aux bibliothèques, aux installations sportives, cours d'anglais,...). 12 enseignes ont recours à ce type de convention signée, dans trois quart des cas, avec des universités. Sur ce point, on peut faire l'hypothèse que ces dernières donnent lieu à une rémunération des écoles d'Etat et des universités. Les investigations sont à poursuivre dans ce domaine. Enfin, un peu moins d'un cinquième des enseignes (12) est reconnu par décret par le ministère de l'Education nationale ou celui de l'Enseignement supérieur

Les enseignes font également massivement référence au RNCP dans la présentation de leurs diplômes (tableau 4). Les deux tiers des enseignes étudiées ici (43) délivrent des certifications inscrites aux niveaux 1, 2 et/ou 3 de ce répertoire. Duke Street met systématiquement en place ce genre de stratégie relativement délaissée par Apax. Sur les sites internet des établissements dont les certifications sont répertoriées, l'inscription est surtout présentée (dans 35 des 43 cas) comme une " certification ", une " homologation" ou une "reconnaissance" "de l'Etat». Les enseignes jouent ainsi sur l'ambiguïté de la procédure et sur des équivalences de diplômes de moins en moins planifiées par la Commission nationale (Maillard et Veneau, 2008). Dans leur publicité, elles font

18. Seul Duke Street ne possède aucune enseigne présentant cette caractéristique.

19. Les écoles de commerce délivraient auparavant le diplôme d'Etat d'études commerciales, créé aprèsguerre (Blanchard, op. cit.).

20. Dans son article 5, La loi Faure (n68-978) de 1968 a en effet autorisé les établissements publics à "passer des conventions de coopération avec d'autres établissements publics ou privés " "notamment [...] [afin] d'étendre aux étudiants des établissements privés les modalités de vérification des aptitudes et des connaissances prévues pour ceux des établissements [...] publics". 
notamment de l'inscription au RNCP un droit de certification similaire à celui dont bénéficient les institutions de l'Etat.

L'examen des textes législatifs français et les usages qu'en font les groupes à but lucratif révèlent ainsi l'existence, avec la globalisation, d'un continuum de gages de qualité plus ou moins accessibles. En France, les établissements de statut privé peuvent notamment bénéficier de certains pouvoirs publics de certification.

Dans les faits, les établissements à but lucratif ont plutôt tendance à délaisser les accréditations d'agence internationale, les reconnaissances d'établissement et les autorisations de délivrer des "diplômes nationaux ", des dispositifs dont on peut faire l'hypothèse qu'ils sont encore trop centrés sur des exigences de qualification du personnel et/ou de programmes pour que les groupes à but lucratif puissent ou veuillent les mobiliser.

Au sein de quatre des cinq groupes étudiés, on observe ainsi que quelques écoles délivrent le grade de master ou le titre d'ingénieur, tandis que les autres délivrent des certifications non référencées ou simplement inscrites au RNCP. Le succès du RNCP et dans une moindre mesure, celui des préparations aux concours de comptabilité et de techniciens supérieurs pourraient alors s'expliquer par les qualités différentes qu'ils présentent. Le RNCP est centré sur des exigences d'insertion professionnelle. Les préparations aux diplômes de comptabilité et de techniciens comportent plus de garanties sur les conditions de préparation aux épreuves du diplôme national que sur son obtention effective par le candidat inscrit dans le secteur privé (MEN, 2013 ; p. 20). Ces hypothèses contribueraient également à expliquer la référence systématique aux certifications de l'architecture européenne des diplômes (LMD - licencemaster-doctorat) et aux intitulés des diplômes délivrés par les universités britanniques et américaines, des reprises rendues possibles par la loi sans qu'elles ne renvoient à une quelconque convention entre établissements. La quasi-totalité des établissements délivrent des "bachelors" et des Master of business administration $(M B A)$ - le diplôme international de référence en matière d'administration des affaires économiques ; seulement un cinquième des enseignes fait en effet l'impasse sur ces deux intitulés. Par ailleurs, les intitulés sont présentés dans les termes du Processus de Bologne et des décrets d'application du LMD. Tout en prenant quasi systématiquement la précaution de ne pas les assimiler à une "licence " ou un " master ", appellations protégées par la loi française, les établissements du panel présentent leurs bachelors et leur $M B A$ comme des ensembles de 60, 120 ou 180 "ECTS ", les unités capitalisables d'enseignement dont sont composés les diplômes du LMD. Ce type de références et les intitulés de diplômes qui y sont associés se renforcent parfois lorsque sont évoquées les implantations à l'étranger des enseignes (en Europe occidentale, dans la majorité des quatorze cas d'implantation recensés) et les échanges auxquels elles peuvent donner lieu pour les étudiants. 


\section{Conclusion}

L'enseignement supérieur à but lucratif occupe encore une place mineure dans le paysage français. Son étude n'est toutefois pas sans enseignements pour qui souhaite explorer les nouvelles divisions de l'enseignement supérieur international, marquées par la dissolution des frontières public/privé et la spécialisation des établissements sur des domaines où leurs qualités sont établies par des tiers et où ils démontrent leur rentabilité économique. Plus qu'un secteur aux frontières bien délimitées, les groupes d'établissements cherchant à valoriser leur capital rassemblent, en effet, des écoles de nature différente et qui évoluent dans un flou institutionnel, juridique et financier tenant notamment aux législations existantes et à l'entremêlement qu'elles instituent.

Dans un contexte de globalisation de l'enseignement supérieur, les résultats de l'enquête révèlent, premièrement, que l'enseignement supérieur à but lucratif se finance, se qualifie et se légitime à partir des porosités public/privé induites ou tolérées par les législations existant, en France, depuis plusieurs décennies. L'essor de ces groupes reposerait ainsi moins sur un effacement de la puissance publique que sur la capacité des enseignements technique et libre à mobiliser cette dernière pour servir des intérêts particuliers.

Deuxièmement, si le secteur à but lucratif (et peut-être avec lui, l'ensemble du secteur privé) se propose ici de dispenser, un peu à la manière du public, des enseignements de nature disciplinaire variée, au nom de "valeurs » auxquelles il dit tenir, il présente aussi des dynamiques de différenciation et de hiérarchisation intra-sectorielle encore peu établies dans les universités publiques. Tandis que ces dernières délivrent encore indistinctement, malgré la recherche de "pôles d'excellence " (Pôles de recherche et d'enseignement supérieur, ...), licence, master et doctorat ${ }^{21}$, les établissements à but lucratif semblent, sur ce plan, se distinguer. Au vu des hiérarchies internes des groupes ou de la concentration du groupe Duke Street sur des formations inscrites au RNCP, on peut en effet faire l'hypothèse que ce sous-secteur a tendance à se polariser : d'un côté, des établissements ayant des ambitions de recherche délivrent des masters ; de l'autre, des écoles relevant de niches spécialisées et délaissées par les établissements publics se contentent de délivrer des certifications préparant les étudiants à un certain type d'emploi.

\section{Bibliographie}

Archer M.S. (1979), Social Origins of educational systems, Sage.

21. En moyenne, en 2012/2013, 60 \% des étudiants des universités sont inscrits en licence (MEN, 2014 ; p. 179). Sur les 75 établissements recensés, l'université de Mayotte (719 étudiants) est le seul établissement à n'inscrire ses étudiants qu'en licence. A l'autre extrême, on retrouve l'université Paris VII (24 443 inscrits) avec 45,8 \% d'étudiants en licence. 
Ball S. (2012), Global education inc. New policy networks and the neo-liberal imaginary, Routledge.

Barber M., Donnelly K., Rizvi S. (2013), An Avalanche is Coming. Higher Education and the Revolution Ahead, Rapport, Institute for Policy Research.

Baudry B. (2003), Economie de la firme, La Découverte, Repères.

Blanchard M. (2012), Socio-histoire d'une entreprise éducative : le développement des Ecoles supérieures de commerce en France (XIXe siècle - 2010), Thèse pour l'obtention du doctorat de sociologie, sous la direction de Beaud S. et Grelon A., EHESS, 23 octobre.

Charle C. et Verger J. (1994), Histoire des universités, PUF, Paris.

Deer C. (2002), Higher education in England and France since the 1980's, Oxford, Symposium Books.

Dubar C. (2004), La formation professionnelle continue, La Découverte, Repères, 5ème édition.

Elledge J. (2014), "Fresh developments", EducationInvestor, vol. 6, n 2, mars, pp. 34-35.

Friot B. (2012), L'enjeu du salaire, La Dispute.

Gadrey J. (2003), Socio-économie des services, La Découverte.

Garcia S. (2008), "L'expert et le profane : qui est juge de la qualité universitaire ?", Genèses, n 70 , pp. 66-87.

Grelon A. (sous la dir.) (1986), Les ingénieurs de la crise : titre et profession entre les deux guerres, Editions de l'école des hautes études en sciences sociales, Paris.

Karpik L. (2007), L'économie des singularités, Gallimard, Bibliothèque des sciences humaines.

Kinser K. and Levy D. (2005), “The for-profit sector: U.S. patterns and international echoes in higher education", PROPHE working paper series, $\mathrm{n}^{\circ} 5$.

Legois J.-P., Monchablon A. et Morder R. (2007) (sous la dir.), Cent ans de mouvement étudiant, Editions Syllepse.

Lewandowski J.-C. (2014), «Un nouveau label de l'Etat pour l'enseignement supérieur privé ", Educpros, 30 juin.

Maillard F. et Veneau P. (2008), «Les niveaux de formation à l'heure européenne : un examen à partir de l'homologation des titres ", Formation Emploi, $\mathrm{n}^{\circ} 102$, avril-juin, La Documentation française.

Ministère de l'Education nationale (2013), Rapport du médiateur de l'éducation nationale et de l'enseignement supérieur. Année 2012, La Documentation française. 
Ministère de l'Education nationale (2014), Repères et références statistiques sur les enseignements, la formation et la recherche, ministère de l'Education nationale et ministère de l'Enseignement supérieur et de la Recherche, Direction de l'évaluation, de la prospective et de la performance, Paris.

Monod O. (2011), «Enseignement supérieur privé : pourquoi les fonds anglo-saxons investissent en France ? ", Educpros, 28 janvier.

Pierru F. (2012), « Le mandarin, le gestionnaire et le consultant. Le tournant néo-libéral de la politique hospitalière ", Actes de la recherche en sciences sociales, vol. 4, n¹94, pp. $32-51$.

Presidential Innovation Lab (2013a), "Unbundling versus designing faculty roles", Presidential Innovation Lab white paper series, American council of higher education.

Presidential Innovation Lab (2013b), "Beyond the inflection point: reimagining business models for higher education”, Presidential Innovation Lab white paper series, American council of higher education.

Vatin F. (dir.) (2009), Evaluer et valoriser : du travail au produit. Une sociologie économique de la mesure, PUM, Toulouse.

Vinokur A. (2004), « Public, privé, ... ou hybride ?, L'effacement des frontières en éducation ", Cahiers de la recherche sur l'éducation et les savoirs, $\mathrm{n}^{\circ} 3$. 